\title{
PORPHYRIA AS A PROBLEM IN ANAESTHESIA*
}

\author{
F. F. LEPINSKIE, M.D., C.M.†
}

Porphyria is a discouraging disease. Even the most skilfully administered anaesthetic may have a fatal outcome in a porphyric, if it includes a barbiturate. It is also an uncommon disease. In the 50 years since it was described, $\mid$ Watson $^{1}$ collected 275 cases of all types occurring in the U.S.A. But the barbiturates are one of the most commonly used groups of drugs in medicine and it is very difficult, today, for any patient to avoid exposure to them. The anaesthetist gives a barbiturate to almost every patient he sees and some/knowledge of this disease is important to him if trouble is to be avoided. Just how important may be illustrated by the following facts.

In Great Britain during the years 1948-53, 32 patients with acute intermittent porphyria were reported ${ }^{2}$; of these, 13 had received thiopentone, all of the 13 developing parralysis, and 5 having a fatal outcome. Again in 1962, Dundee et al. ${ }^{3}$ reported 3 cases of acute intermittent porphyria who were given thiopentone; all were paralysed, two died, and the third made a slow recovery after a year of physiotherapy. The maxim "primum non nocere" seems to be very applicable here.

\section{Ponphyria, Porphyrlugria, and the Póprifyrin Pigments}

The terminology of the porphyrins is apt to be confusing to someone making their acquaintance for the first time. Porphyrin is the Greek word for "purple" and is the name first given to the purple iron-free compound which appears when blood is treated with concentrated $\mathbf{H}_{2} \mathrm{SO}_{4}$. This is a "laboratory porphyrin" and does not occur in nature. It was originally called haematoporaphyrin because of its origin and its colour. Porphyrins are very basically pigments. These pigments are found at all levels of biological life and the most important of them all is haemoglobin. Haemoglobin consists of a protein, globin, united with a porphyrin pigment, heme (heme is iron-protoporphyrin III).

The porphyrin nucleus consists of four pyrolle rings joined together by four "methene bridges" to form the structure shown in Figure 1.

The porphyrins are readily synthesized in the body to take part in the formation of haemoglobin. They are synthesized from acetate and glycine (in the tricarboxylic acid cycle), through a long, complex series of biochemical reactions to porphobilinogen. The porphobilinogen molecules condense to form a four-ringed complex called uroporphyrin (stereo-isomers types I and III may be found). This in turn is converted to coproporphyrin, thence to protoporphyrin-the type III isomer of which is combined with iron and globin to form haemoglobin (Fig. 2).

When haemoglobin is broken down in the body it forms two portions: the one

*Presented at the weekly Geigy meeting of the McGill Diploma Course, February, 1963.

†St. Mary's Hospital, Montreal. P.Q. 


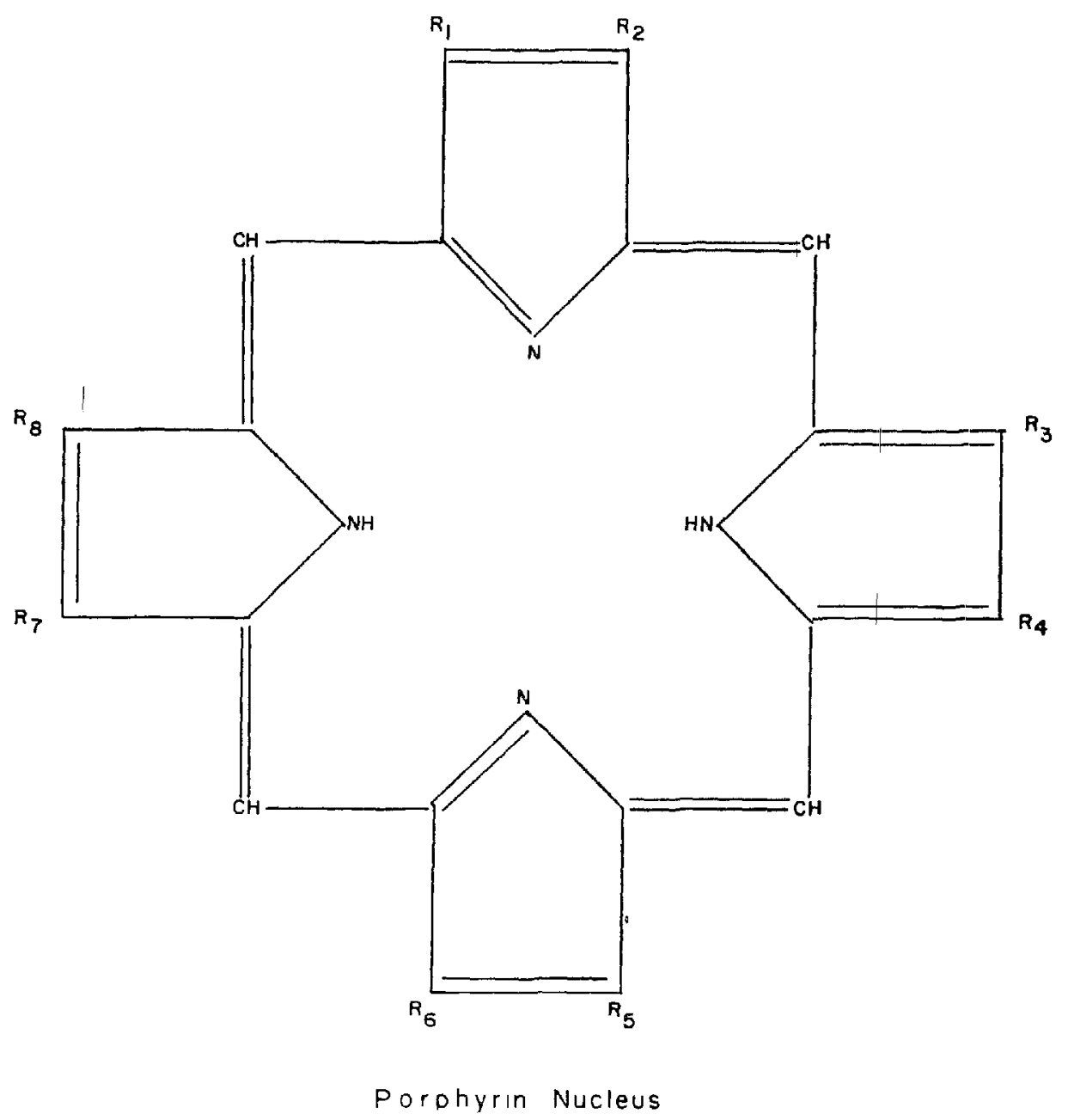

Figure 1.

is iron-free and is called haematoidin; the other is haemosiderin, which contains iron and is retained in the body to be built up again into haemoglobin. The ironfree haemotoidin is converted into bilirubin and excreted in the bile.

Protoporphyrin, as the name suggests, is the prototype of the naturally occurring porphyrins-being the basic pigment, in combination with iron, of the heme of haemoglobin. This compound does not appear in the urine-unlike the other porphyrin pigments-but does appear in faeces, the amount present being related to the amount of blood in the gastro-intestinal tract and to the rate of liberation of porphyrin from haemoglobin by faecal bacteria.

Porphobilinogen is the colourless precursor of the three porphyrins (copro-, uro-, and proto-). It is the one most important to the anaesthetist and will be discussed later.

Uroporphyrin is a red-coloured pigment excreted by the kidney. Normally there are only minute trace amounts in the urine, but large amounts may be present giving the urine a "port-wine" colour. When it is left to stand, the colourless porphobilinogen becomes changed into a red-coloured compound, the structure of which is not known for certain. 

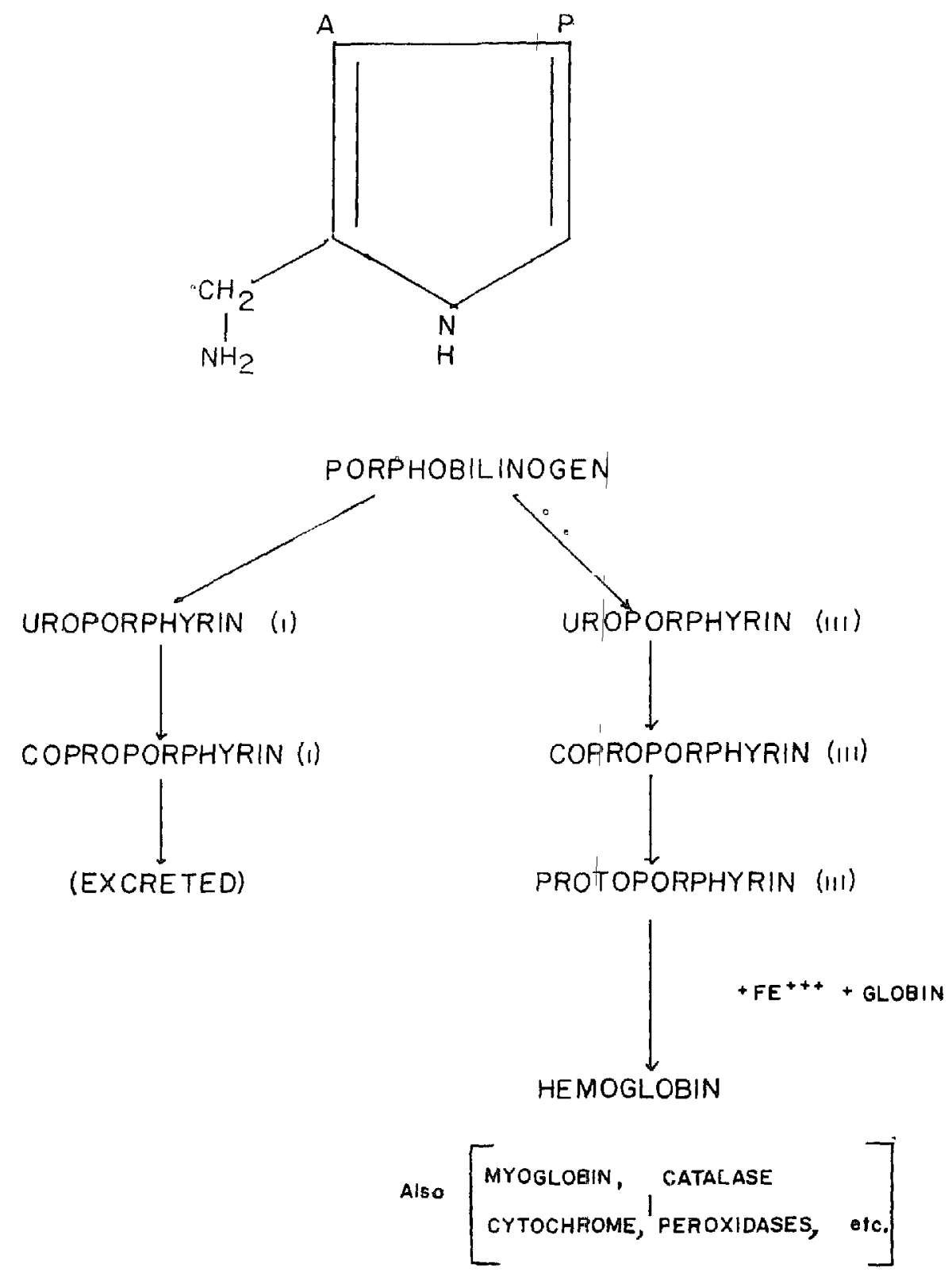

Figure 2.

Coproporphyrin is the porphyrin excreted by the liver into the bile and faeces. ( $k o p r o s=$ dung.) However, it may appear in the urine in certain diseases-the porphyrias and the porphyrinurias.

The term porphyrinuria refers to the excessive excretion of porphyrins in the urine. Such conditions as heavy-metal poisoning, poliomyelitis, Hodgkin's disease, haemolytic anaemias, and drug reactions (mainly to barbiturates and sulphas) are accompanied by the presence of copro- and uro-porphyrins in the urine. In all of these conditions, the increased porphyrin excretion is merely an interesting accompaniment of the disease and does not appear to be an abnormality of porphyrin metabolism.

The term porphyria, however, refers to a spècific group of disease entities 

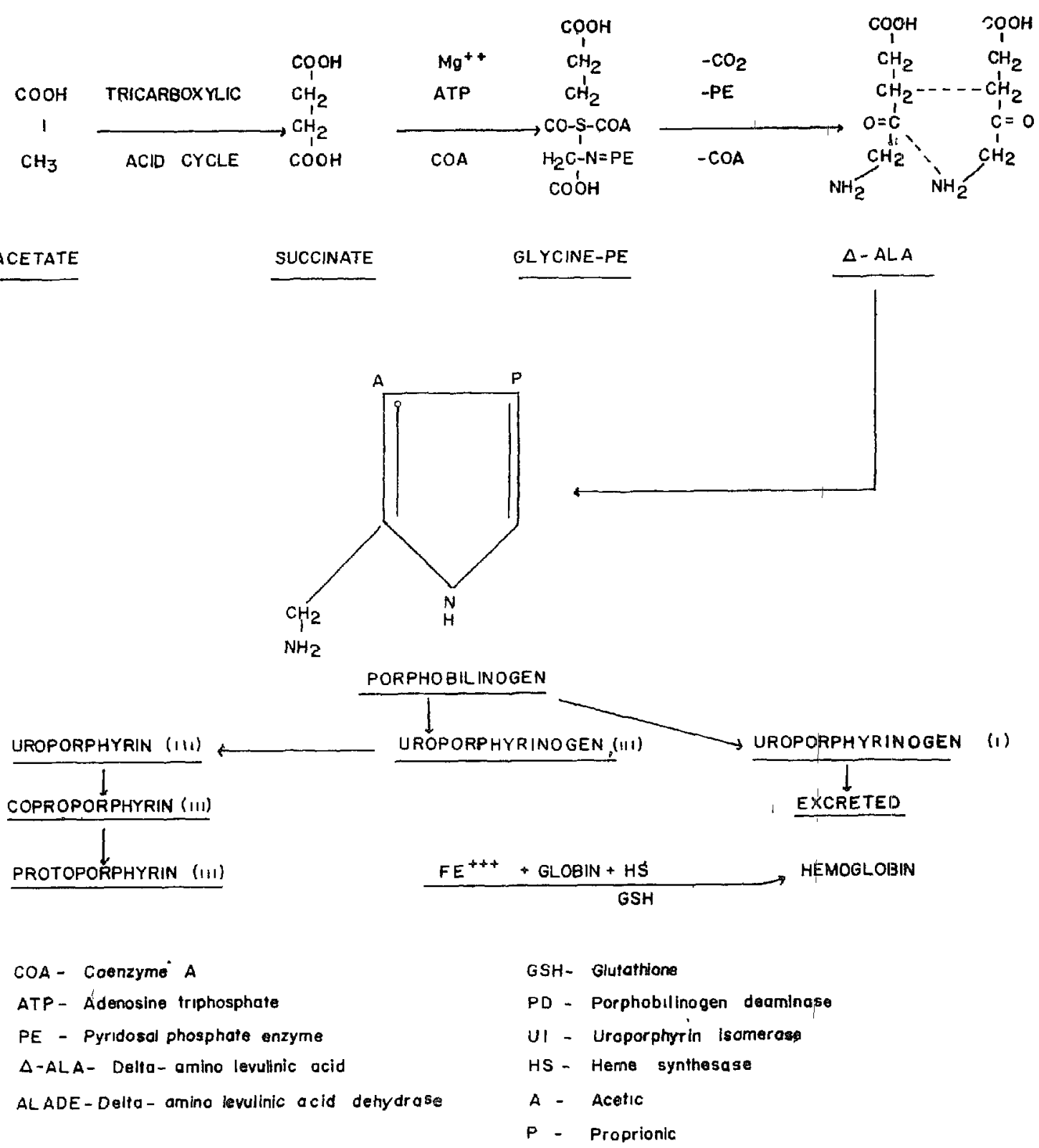

Figure 3.

resulting from an abnormality of porphyrin metabolism. Each has unusual and characteristic manifestations, but all are marked by the excessive excretion of porphyrin pigments and/or their precursors in the urine and/or faeces.

\section{Classification of Porphyrias}

There is no classification of porphyrias agreeable to all. Each author has his own classification, which seems totally unacceptable to most other authors. The one used here is given by Harrison in the latest edition (1962) of his Principles of Internal Medicine ${ }^{4}$ :

I. Porphyria erythropoietica: congenital porphyria. 
II. Porphyria hepatica:

(a) Acute intermittent porphyria ("AIP"), acute or latent.

(b) Porphyria cutanea tarda hereditaria, acute or latent.

(c) Porphyria cutanea tarda symptomatica.

(d) Unclassified.

\section{Description of Disease}

\section{Porphyria Erythropoietica}

This, as the name implies, consists of a basic porphyrin defect in the erythropoietic system and large quantities of porphyrins are present in the normoblasts (the uroporphyrins imparting a red hue to the skeletal structures). This is one of the rarest of human diseases-only 34 authentieated cases could be found since the original description by Gunther in $1911 .{ }^{4}$ It is a congenital disease and hence present from birth. It is characterized by red urine, red skeleton and teeth, photosensitivity of the skin, repeated infections, a haemolytic anaemia, and splenomegaly. There are no central nervous system disturbances and no abdominal symptoms; porphobilinogen is not present in the urine, barbiturates do not affect the course of the disease, which is progressively downhill. Death is usually due to repeated infections of skin lesions and bronchopulmonary system.

\section{Porphyria Hepatica}

This next group of porphyrias is so called because the site of storage of the abnormal porphyrms is not in the bone marrow but in the liver. By far the most common and the most serious form of the disease is called "Acute Intermittent Porphyria" ("AIP"). Because it is so important to us, it will be dealt with at some length.

The disease is essentially an inborn error of porphyrin metabolism affecting the central nervous system and is inherited through a non-sex-linked Mendelian dominant gene. It affects both sexes but women are more often the victims. The onset is usually after puberty and before the fourth decade. Although it is an uncommon affliction, it is by no means rare. Of a total of 275 cases collected in the U.S.A. up to 1954, Watson found that 199 were of the acute intermittent type and only seven were congenital. ${ }^{1}$

"AIP" exists in two forms-acute and latent The latent form is so named because there are no signs or symptoms-indeed the only abnormality is the presence of porphobilinogen in the urine. Just how this latent form can be converted into the full-blown acute phase is still a subject of much argument. Drugs such as barbiturates, chloroquine, progesterone, ethyl alcohol, certain sulphas, and lead are known to have this effect. Pregnancy and menstruation are said to have an adverse effect, but the opposite can occur, and why this should be so remains a mystery.

The clinical picture of the acute phase is a complex one and depends on which parts of the nervous system are affected. The symptoms are abdominal, neurological, and psychic or many be any mixture of the three. ${ }^{5}$ The classical picture is that of an acute surgical abdomen with severe crampy abdominal pain, accom- 
panied by vomiting, diarrhoea or constipation, fever, tachycardia, and leukocytosis. Naturally enough it is commonly mistaken for appendicitis, pancreatitis, cholecystitis, or renal colic. There are, however, other symptoms which add to the confusion. In order of frequency they are muscle weakness ( 75 per cent), mental changes such as hysteria, hallucinations, depression, and sometimes coma (60 per cent), diminished or absent reflexes ( 50 per cent), deep muscle pain and sensory changes ( 30 per cent), and cranial nerve palsies ( 30 per cent). The urine is red (due to uroporphyrins) in 60 per cent of cases; some 50 per cent have a significant degree of hypertension during the whole course of the disease. The tachycardia, however, remains only during the acute episode and reverts to normal with each remission. All, however, have porphobilinogen in their urine, in large amounts during an acute episode, and once this has been found, the search is over.

But the diagnosis is never easy and even the best are misled by the fact that "common things are commonest." The abdominal picture can mimic any acute abdomen; but a long-standing history of intermittent abdominal colic for which previous laparotomies have been ineffectual should make one suspicious. A family history of similar bizarre episodes or unusual reaction to barbiturates as well as the statement that red or black urine has been voided on previous occasions should prompt a search for porphobilinogen. This is often a disease of long standing with episodes of remission and relapse but once neurological symptoms occur, the prognosis changes from bad to worse. Death may occur in two ways, either from respiratory paralysis or from intractable diarrhoea with massive fluid loss and a hypokalaemic açidosis going on to coma and death.

\section{Pathology}

There is a profound, basic disturbance in porphyrin metabolism, resulting in withdrawal and excretion of abnormal amounts of metabolites from this system. This in turn is related to the neurological lesions, but how and where this disturbance occurs and how this in turn affects the metabolism of myelin is unknown. The principle neurological lesion is one of demyelination of the neural sheaths and, eventually, Wallerian degeneration. This occurs throughout the entire central nervous system in a scattered, haphazard fashion, but is usually a late feature of this disease. In other words, symptoms appear long before the neurological lesions can be found to explain them. Another late finding is destruction of the nerve cells themselves. This is said to occur at all levels, from the cord to the cerebral cortex. Why this occurs is still unexplained but it is known that porphyrins are not increased around areas of degeneration, so demyelination is probably not directly due to these substances.

It has been suggested ${ }^{B}$ that zinc, or some other heavy metal, could be responsible for an enzymatic block in the synthesis of porphyrin and that removing this metal with a chelating agent ${ }^{*}$ could help correct the metabolic error. Increased

A chelating agent is one that will neutralize the effect of a heavy metal. It is usually a rung-shaped structure that will form a loose combmation with the metal, binding it and removing it from the body in an inactive form. 
urinary levels of zinc and lead during an acute episode seem to support this idea -as well as the fact that both zinc and lead can produce a clinical picture somewhat similar to "AIP" with abdominal colic and neuropathy. It has been found that zinc and lead levels in the urine have been markedly raised while an acute porphyric was being treated with chelating agents, suggesting that chelation was rapidly neutralizing the heavy metal by binding it and removing it from vulnerable enzyme systems.

The demonstration of the porphobilinogen in the urine is necessary before the diagnosis of "AIP" can be confirmed. Porphobilinogen is colourless:and when freshly voided urine is examined, it will be normal in colour (unless other porphyrins are present, such as uroporphyrin or coproporphyrin). The "port wine" urine of porphyria is actually due to uroporphyrin and not to the colourless monopyrrole. The presence of porphobilinogen-can be demonstrated in three ways:

1. By leaving the urine in sunlight for a few hours; there is a definite deepening of colour compared with the same specimen kept in the dark.

2. By heating the same urine in a water bath for 10 minutes and comparing the colour with that of an unheated specimen; the heated urine turns dark.

- 3. By performing the Watson-Schwartz test for porphobilinogen ${ }^{T, 8,9}$ if laboratory facilities are available: 2 c.c. of urine and 2 c.c. of Erlich's aldehyde reagent are mixed and allowed to stand for 2 minutes. If porphobilinogen or urobilinogen is present, a red-coloured compound is formed. Which of the two is present can be distinguished by the addition of 4 c.c. of chloroform (or amyl alcohol); urobilinogen dissolves in the alcohol or chloroform while porphobilinogen remains in the aqueous solution.

\section{TrEATMENT}

Many agents have been used in the treatment of an acute attack but spontaneous 'remission is part of the natural course of the disease and results are therefore difficult to interpret.

The abdominal symptoms are usually well controlled by chlorpromazine ${ }^{10}$, others $^{11}$ have used $d$-tubocurare $(0.15 \mathrm{mg}$. $/ \mathrm{kg}$. $)$, tetraethyl ammonium chloride. priscoline, nitroglycerin, and neostigmine. ${ }^{12,13}$ Blocking the celiac ganglion has been tried and vagotomy has been suggested.

The steroids ${ }^{6}$ have been used along with ACTH but, on the whole, results have been disappointing.

As mentioned previously," chelating agents (BAL and EDTA $\dagger$ ) have been used in the management of acute episodes with apparently good results, and this would seem to be one of the most promising approaches for further study. But from the multitude of suggested remedies, it would seem that there is no good single agent available yet. Therefore prophylaxis is of great importance here. The agents capable of precipitating an acute attack should be avoided. This includes all the barbiturates, the sulpha drugs, alcohol, heavy metals and oil-based paints.

${ }^{*} \mathrm{BAL}$ is British Anti-lewisite, given 50-1200 mg./day in divided doses.

TEDTA is disodium calcium ethylene diamine tetra-acetate, given $1-10 \mathrm{gm} . / 24 \mathrm{hr}$. 
chloroquine, and progesterone. Pr egnancy may precipitate an acute episode, but paradoxically, some porphyrics enjoy their best health when pregnant and its prevention is not always easy.

The over-all prognosis is poor. Durng an acute episode, the mortality rate in some series is as high as 50 per cent. However, the high mortality is usually due to respiratory paralysis, and if active treatment in a respiratory-failure unit wére used, there is reason to believe that these figures could be reduced It has also been stated that the damage to the myelin sheath is reversible and, if so, maintenance in a respiratory unit for a prolonged period of time may prove worth while. The merit of these ideas will have to be confirmed with further work, because it is not generally accepted that the changes are teversible-though function in the damaged areas may return temporarily.

\section{Other Forms of Porphyria}

\section{Porphyria Cutanea Tarda Hereditaria}

This is a cutaneous form of porphyria occurring in later life. In some ways it is analogous to congenital porphyria; it is inherited and is marked by skin lesions. In this condition, however, the skin lesions are not as mutilating as in the infantbut they are bullous or blistering in type and show a marked photosensitivity for the sun's actinic rays. The skin's unusual sensitivity is said to be due to abnormal porphyrin pigments in the skin itself.

Porphyria cutanea tarda hereditaria occurs in two forms, ${ }^{\mid}$acute and latent. Along with skin lesions, the acute type may have central nervous system and abdominal symptoms, indistinguishable from acute intermittent porphyria, along with the appearance of porphobilinogen in the urine. Coproporphyrins and uroporphyrins are also present, in large amounts, in the faeces at all times, porphobilinogen appearing only during the acute episodes. For this reason, it has been suggested $^{14}$ that as long as the liver can handle large amounts of porphyrins and excrete them as bile, the disease remains latent; once the liver cannot handle the extra work load, porphobilinogen appears in the urine and an acute attack occurs. For all practical purposes this can be considered as the same disease as "AIP"with skin lesions.

The only abnormality found in the latent form is abnormal amounts of coproporphyrin in the faeces at all times. There is no porphobilinogen in the urine during the latent phase and urinary coproporphyrins and uroporphyrins are in the normal range.

\section{Porphyria Cutanea Tarda Symptomatica}

Unlike all the other porphyrias, this is an acquired form of the disease. It occurs at all ages, in both sexes, and is marked by cutaneous lesions (identical with those of cutanea tarda), hyperpigmentation, hypertrichosis, and evidence of liver disease with large amounts of uroporphyrins and coproporphyrins in the urine. Faecal porphyrins are in normal quantity and there is a normal amount of porpholilinogen in the urine. There are no abdominal or central nervous system symptoms. This form of porphyria has been reported ${ }^{4}$ as occurring in: (1) males 
aged 40-70 years with alcoholic cirrhosis, (2) South African natives with nutritional cirrhosis, (3) Turkish farmers after the ingestion of hexachlorobenzene, (4) three adults with liver tumours (hepartomas) and one ovarian teratoma, (5) young adults with a history of alcohol or drug exposure.

The Turkish farmers probably represent the first acquired toxic form of porphyria in man. In 1955 Schmid $^{15}$ went to Turkey to investigate the unusual skin disease that had suddenly appeared. He found approximately 5000 people with cutaneous bullae, red urine (uroporphyrins), hypertrichosis, and hepatomegaly. There were no abdominal or central nervous system complications and no porphobilinogen in the urine. All of these people had eaten wheat grown from seed that had been treated with the fungicide hexachlorobenzene.

The principal clinical characteristics of the whole group 'may now be summarized as shown in Table I ( from Harrison ${ }^{4}$ ).

TABLE I

\begin{tabular}{|c|c|c|c|c|c|c|}
\hline Characteristic & Congenital & \multicolumn{2}{|c|}{ AIP } & \multicolumn{2}{|c|}{ PCT (Hered.) } & PCT (Symp.) \\
\hline Inheritance & Recessive & \multicolumn{2}{|c|}{ Dominant } & \multicolumn{2}{|c|}{ Dominant } & Acquired \\
\hline Sex & - & \multicolumn{2}{|c|}{$q>\delta$} & \multicolumn{2}{|c|}{$\hat{\sigma}>0$} & Both \\
\hline Age of onset & $0-5$ & \multicolumn{2}{|c|}{$15-40$} & \multicolumn{2}{|c|}{$10-30$} & Any \\
\hline Phase of disease & - & Latent & Acute & Latent & Acute & \\
\hline Cutaneous lesions & $++t+$ & - & - & - & + & + \\
\hline Abd. colic CNS & 0 & 0 & + & 0 & + & 0 \\
\hline \multicolumn{7}{|l|}{ Urine } \\
\hline Porphobilinogen & $\mathrm{N}$ & + or $N$ & $+t+$ & $\mathrm{N}$ & $+t$ & $\mathbf{N}$ \\
\hline Uroporphyrin & $+t+t$ & ++ & ++ & $\mathrm{N}$ & ++ & $+t+t$ \\
\hline Coproporphyrin & ++ & ++ & $+t$ & $\mathrm{~N}$ & $+t+$ & ++ \\
\hline Faecal porphyrins & +++ & $\mathrm{N}$ & + & $+t+t$ & $t+t$ & $\mathrm{~N}$ \\
\hline Barbiturate effect & - & - & +++ & - & $++t$ & - \\
\hline
\end{tabular}

Anaesthetic Management

\section{Preoperative Assessment}

When confronted with an acute porphyric for anaesthesia, besides the usual examination and questioning, four particular facets of the patient's condition should be examined rather thoroughly. They are: (1) neurological changes, (2) electrolyte changes, (3) cardiovascular changes, and (4) respiratory system changes. The patient should be carefully questioned about neurological symptoms; the lesions, if present, should be mapped out and recorded on the chart so that subsequent changes may be compared with the preoperative status. Electrolyte changes are to be expected if abdominal symptoms have been prolonged and accompanied by vomiting or diarrhoea. Hypochloraemic alkalosis is expected after the former and hypokalaemic acidosis after the latter. The blood pressure and pulse are of interest and both are usually elevated during an acute episode. The hypertension is quite labile and may fall abruptly once anaesthesia has commenced and the normal protective teflexes have been obtunded. A rough "working estimate" of respiratory reserve can be obtained from the history and the simpler tests, such as timed vital capacity and maximum breathing capacity, can be done if indicated, as well as blood gas studies. At the very best of times, 
these patients are usually considered as a "calculated risk"|from 'the anaesthetist's point of view. ${ }^{16}$

Premedication. A barbiturate should never be given to a patient suspected of having porphyria. All the other ptemedicant drugs have been used and chlorol hydrate would be an admirable substitute for the usual hypnotic. The phenothiazine drugs have been used to great advantage here-particularly promethazine. The patient's chart should be clearly marked in large letters on the cover, "NO BARBITURATES," lest some solicitous attendant quieten the restless poirphyric with "a little sedative."

Anaesthetic agents. Adl of the inhalation agents are quite safe and any may be used. Induction, of course, is accomplished with an inhalation agent.

Relaxants. Succinylcholine is regarded as the relaxant of choice; but all have been used without adverse effects. The preference is more theoretical than pragmatical. It is known that certain phosphorus-containing insecticides with anticholinesterase activity can produce demyelination and therefore other anticholinesterase agents should be avoided on theoretical grounds. On the other hand, neostigmine and curare have been used in the past to treat acute attacks without apparent harm. But in England, at least, the non-depolarizing relaxants are not used for this reason.

Conduction anaesthesia. It seems unwise to use this form of anaesthesia, unless forced by circumstances to do so. Although local' anaesthetic agents act at "nodes of Ranvier," some must penetrate the myelin sheath. The characteristic lesion of this disease is demyelination and to interfere in this area, unless forced to do so, would seem to be begging trouble. Pareses and paralyses can occur at any time in "AIP," but their occurrence after a regional block (spinal, epidural, or local infiltration) would surely invite litigation. General anaesthesia offers a wide choice of agents and conditions can be made just as suitable for the surgeon; therefore, unless contraindicated for some other reason, general anaesthesia should be used.

\section{Postoperative Management}

Any of the conventional narcotic analgesics can be used to control pain. The ataractic phienothiazines may be used as a supplement, since chlorpromazine has been used to treat the acute symptoms of this condition. To date, there is no report in the literature on the use of the synthetic hypnotics such as glutethamide, and for the present they are better avoided.

\section{SUMAIARY}

Porphyria represents a basic metabolic disturbance and appears in many forms. Two of these, both -acute, are of importance to the anaesthesiologist. It is a difficult disease to recognize because it can simulate so many other abdominal emergencies. When confronted with these emergencies, only an awareness of the disease and a knowledge of its intricacies will suggest the possibility that this condition is present and lead to testing of the urine for porphobilinogen. 
The future treatment of the disease may hold a large role for the anaesthesiologist. Severe neurological involvement with respiratory failure may soon be treated in the respiratory unit and then, perhaps, the high mortality rate can be lowered.

Dundee ${ }^{2}$ feels that this disease is possibly the only single absolute contraindication to using thiopentone as an induction agent. All other anaesthetic agents, with the possible exception of neostigmine and regional techniques, may be employed.

\section{REFERENCES}

1. Watson, C. J. The Problem of Porphyria-Some Facts and Questions. New Eng. J. Med. 263: 1205 (1960).

2. Dundee, J. \& Rming, J. Barbiturate Narcosis in Porphyria. Anaesthesia 10: 55 (1955).

3. Dundee, J.; McCunany, N.; \& McLoughuin, G. The Hazard of Thiopental Anaesthesia in Porphyria. Anesth. \& Analg. 41: 567 (1962):

4. Harrison, T. R. Principles of Internal Medicine, p. 758. 4th ed. New York: McGraw-Hill (1962).

5. Kark, R. M. Clinical Aspects of the Porphyrias. Med. Clinics of N. America (Jan. 1955).

6. Peters, $H$. et al. The' Treatment of AIP with Chelating Agents. Ann. Int. Med. 47: 889 (1957).

7. - Therapy of Acute, Chronic and. Mixed Hepatic Porphyria with Chelating Agents. Neurology 8: 621 (1958).

8. PĖTÉRS, H. BAL Therapy of Accute Porphyrinuria. Neurology 4: 477 (1954).

9. Watson, C. J. \& Schwartz, S. A Simple Test for Urinary Porphobilinogen. Proc. Roy. Soc. Exptl. Biol. \& Med. 47: 393 (1941).

10. Melby, J. C.; Street, J. P.; \& Watson, C. J. Chlorpromazine in the Treatment of Porphyria. J.A.M.A. 162: 174 (1956).

11. Weriracher, W. H. New Symptomatic Treatment for Acute Intermittent Porphyria. Arch. Int. Med. 89: 111 (1952).

12. Gondin, R. Prostigmin via akut porfyri. Nord. Med. $37: 480$ (1948).

13. Ginlmespy, R. \& SMIth, S. G. Porphyria Treated with Neostigmine. Lancet 266: 908 (1954).

14. Waldenström, J. The Porphyrias as Inborn Errors of Metabolism. Am. J.,Med. 22: 758 (1957).

15. Scrmm, R. Cutaneous Porphyria in Turkey. New Eng. J. Med. 263: 397 (1960).

16. Nonris, W. \& MCNAB, G. W. Anaesthesia in Porphyria. Brit. J. Anaesth. 32: 505 (1960). 